\title{
Mortalidade de adolescentes em área urbana da região Sudeste do Brasil, 1984-1993
}

\author{
Adolescent mortality in an urban area of Southeastern Brazil, \\ 1984-1993
}

\author{
Sílvia M. Kawata Lyra, Tamara Goldberg e Massako lyda \\ Departamento de Pediatria da Faculdade de Medicina da Universidade Estadual Paulista. \\ Botucatu, SP - Brasil (S.M.K., T.G.), Departamento de Saúde Pública da Faculdade de Medicina \\ da Universidade do Estado de São Paulo. Botucatu, SP - Brasil (M.I.)
}

\begin{abstract}
Resumo
Analisa-se a mortalidade de adolescentes no Município de Botucatu, Estado de São Paulo, Brasil, no período de 1984 a 1993, segundo dois subgrupos (10 a 14 e 15 a 19 anos), sexo, ocupação e causas de óbito. Os dados de óbitos foram obtidos no Setor de Estatística do Centro de Saúde-Escola. As estimativas populacionais foram calculadas com base nos censos demográficos. Observou-se variação dos coeficientes de mortalidade nos diferentes anos e maior mortalidade no grupo masculino de 15 a 19 anos, atingindo tanto estudantes como trabalhadores. Houve predomínio de causas externas de mortalidade, principalmente acidentes de trânsito e ferimento por arma de fogo, exigindo averigüação de seus determinantes e o desenvolvimento de programas de saúde destinados aos adolescentes, suas famílias e à sociedade, considerando-se que as causas de morte são evitáveis e preveníveis.
\end{abstract}

Mortalidade. Adolescência. Causa de morte.

\begin{abstract}
Mortality among adolescents resident in the municipal district of Botucatu, State of S. Paulo, Brazil, according to age group (10 to 14 and 15 to 19 years), sex, occupation and cause of death, during the period from 1984 to 1993, was analysed. The mortality data were obtained from death certificates and the population estimates were based upon census figures. There was great variation in the mortality rates of the years studied. The mortality was greater among the males of the older age group (15 to 19 years), including both students and workers. There was predominance of external causes of mortality which suggests the need for further studies its determining factors. The data indicate that health programs targeted at regarding adolescents, their families and community should be developed since these are avoidable causes of death.
\end{abstract}

Mortality. Adolescence. Cause of death.

Correspondência para/Correspondence to: Massako Iyda - Departamento de Saúde Pública da Faculdade de Medicina da Universidade Estadual Paulista. Caixa Postal 549 - 18618-970 Botucatu, SP - Brasil. Fax: (014) 822-0421 E-mail: miyda@ fmbunesp.br

Edição subvencionada pela FAPESP. Processo 95/2290-6.

Reccebido em 9.10.1995. Reapresentado em 8.7.1996. Aprovado em 16.7.1996. 


\section{INTRODUÇÃO}

A adolescência é definida como o período de transição entre a infância e a idade adulta, em que ocorrem profundas transformações biopsicosociais. É a fase da vida em que se verificam progressivas alterações físicas, tais como, aumento da massa corporal, aumento da velocidade de crescimento e aparecimento de caracteres sexuais secundários, entre outros. Caracteriza-se, também, por ser a fase em que o indivíduo está em busca de sua própria identidade sendo, assim, um ser contestador, curioso e insatisfeito já que defronta, agora, como seu novo corpo, com novas visões da família e da sociedade, na qual terá um novo papel social a partir de sua escolha sexual e profissional. Somadas a estas características, o adolescente é um ser sem limites, que está, sempre, à procura de novos desafios, impetuoso mas, ao mesmo tempo, imaturo e inseguro.

Pela Organização Mundial de Saúde ${ }^{14}$, a adolescência compreende a fase da vida que vai dos 10 aos 19 anos, subdividindo-a em dois subperíodos: de 10 a 14 anos e de 15 a 19 anos; para o Estatuto da Criança e do Adolescente ${ }^{3}$, esta fase é menor, compreendendo os indivíduos da faixa etária de 12 a 18 anos.

A adolescência, enquanto grupo social, era pouco estudada no Brasil e muitas das razões para sua importância emergente estão relacionadas às extraordinárias mudanças econômicas e sociais, ocorridas no País nos últimos 30 anos. Assistiu-se a um intenso processo de urbanização e industrialização, principalmente, no Estado de São Paulo. Em 1970, neste Estado, 80,4\% da população vivia em áreas urbanas ${ }^{6}$, passando para $88,7 \%$, em $1980^{7}$.

Nesse período de profundas mudanças, o número de adolescentes, no Estado de São Paulo, dobrou, em termos absolutos, passando de 3.049.157, em 1970, para 6.199.985, em 1991. Sua participação no total da população, contudo, decaiu; após elevar-se entre $1970 / 1980$ de $17,2 \%$ a $20,8 \%$, decresceu para $19,6 \%$, em $1991^{5,8,9}$.

No Município de Botucatu, este processo foi mais lento. Em 1960, o número de adolescentes era de 9.778 passando para 12.078 , em 1970, 13.046, em 1980 e 18.188, em 1991, ou seja, a duplicação ocorreu nos últimos 40 anos. A participação dos adolescentes elevou-se de 22,2\% em 1960 para $23,3 \mathrm{em}$ 1970 e manteve-se praticamente estável nas duas décadas posteriores, em torno de $20,0 \%$, diferentemente do Estado. Em Botucatu, com uma população de 64.539 habitantes, em 1980 e de 90.961, em 1991, respectivamente, $13.046(20,2 \%)$ e $18.188(20,0 \%)$ eram adolescentes. A razão de masculinidade no gru- po de 10 a 19 anos foi 1.017 e $1.023 /$ mil mulheres, respectivamente, em 1980 e 1991, havendo, portanto, uma leve predominância do sexo masculino ${ }^{5,8,9}$.

Embora este grupo populacional seja menos vulnerável às doenças, quando comparado com grupos de idades menores, suas condições de sobrevivência têm sido ressaltadas, principalmente, no que se refere ao trabalho e educação. E, embora a taxa de mortalidade, também, seja baixa se comparada com a de outros grupos etários, ela tem se revelado importante, uma vez que resulta de causas evitáveis, num grupo populacional fundamental para renovação de uma sociedade, seja em termos biológicos ou sociais.

Conforme dados da literatura, a mortalidade atinge, com maior intensidade, o grupo de 15 a 19 anos do que o de 10 a 14 anos e, principalmente, o sexo masculino em ambas faixas etárias ${ }^{4}$. Assinala-se, também, que grande parte dos óbitos resulta de causas externas $^{1,2,4,12}$, preponderando os acidentes de trânsito, seguida dos homicídios, com taxa cinco vezes maior no grupo de 15 a 19 anos. Os suicídios aparecem com maior significância no mesmo grupo, sendo três vezes maior do que no grupo de 10 a 14 anos, indicando a importância de fatores vinculados à adolescência ${ }^{4}$.

Dada à importância desse grupo etário e a necessidade de conhecer algumas de suas características, o presente trabalho analisa as principais causas de óbito da população adolescente, no Município de Botucatu, nos últimos dez anos.

\section{METODOLOGIA}

Para o estudo de mortalidade dos adolescentes, no Município de Botucatu, foi utilizada a delimitação etária proposta pela OMS, de 10 a 19 anos, com seus subgrupos.

As causas de óbitos foram coletadas em um centro de saúde-escola, para o período de janeiro de 1984 a dezembro de 1993, num total de 124 óbitos registrados no cartório do município. As causas de óbitos foram codificadas, tendo como base a Classificação Internacional de Doenças $^{13}$. Usou-se a classificação suplementar "E" na qual se incluem os acidentes, quedas, efeitos adversos, suicídios e homicídios ${ }^{11}$.

Os dados populacionais provêm dos Censos Demográficos de $1980^{8}$ e $1991^{9}$. As estimativas populacionais, para 1984 a 1993, foram calculadas pelo método de crescimento aritmético para o meio do período ( $1^{\circ}$ julho). Com base na proporção da participação do grupo etário de 10 a 19 anos, nos censos de 1980 e 1991, foi calculado o número de adolescentes, entre 1984 a 1993.

A partir desses dados, foram obtidos os coeficientes de mortalidade específicos por idade e mortalidade proporcional por causas, segundo diferentes subgrupos. 


\section{RESULTADOS}

Observou-se um crescimento da população de adolescentes, em termos absolutos, passando de 14.735 a 19.026 no período. Os coeficientes de mortalidade mostraram-se bastante variáveis, crescendo entre 1984 a 1986 e em 1989,1990 e 1992, apresentando decréscimo nos anos intermediários. O coeficiente de mortalidade mais baixo entre os adolescentes foi encontrado no início do período, 27,1/ 100.000, passando, em 1993, para 78,8/100.000. Em 1985, esse coeficiente situava-se em um nível intermediário entre os coeficientes apresentados na $\mathrm{Ca}-$ pital do Estado (144,0/100.000) e no Interior $(75,0 /$ 100.000). Bastante expressivos foram os anos de 1990 e 1992, quando os coeficientes elevaram-se para 102,2 e 107,5/100.000, respectivamente (Tabela 1).

Tabela 1 - População, número de óbitos e coeficientes de mortalidade específica de adolescente de 10 a 19 anos, Município de Botucatu (SP), 1984 a 1993.

\begin{tabular}{cccc}
\hline Ano & $N^{0}$ óbitos & $\begin{array}{c}\text { População } \\
10-19 \\
\text { de idade }\end{array}$ & $\begin{array}{c}\text { Coefic/ } \\
100.000\end{array}$ \\
\hline 1984 & 4 & 14.735 & 22,1 \\
1985 & 14 & 15.212 & 92,0 \\
1986 & 13 & 15.689 & 82,8 \\
1987 & 7 & 16.161 & 43,3 \\
1988 & 7 & 16.642 & 42,0 \\
1989 & 10 & 17.119 & 58,4 \\
1990 & 18 & 17.596 & 102,3 \\
$1991 *$ & 16 & 18.188 & 88,0 \\
1992 & 20 & 18.550 & 107,5 \\
1993 & 15 & 19.026 & 78,3 \\
\hline *Fonte: Fundação IBGE', 1991 & &
\end{tabular}

*Fonte: Fundação IBGE ${ }^{9}, 1991$

Do total de 124 óbitos, $63,7 \%$ ocorreram na faixa etária de 15 a 19 anos e $36,3 \%$, entre 10 a 14 anos, confirmando os dados encontrados na literatura (Tabela 2). Fica evidenciada a alta proporção de óbitos devido às causas externas, $70,2 \%$ do total, sendo elevada a proporção no grupo de 15 a 19 anos,
$72,3 \%$ e $27,6 \%$ no grupo de 10 a 14 anos, ocupando o primeiro lugar como principal causa de óbito. Situaram-se em segundo lugar, os neoplasmas (cap.II) da Classificação Internacional de Doenças, com 5,6\% do total de óbitos. Em terceiro lugar, com a mesma proporção, $4,8 \%$ os sinais, sintomas e afecções maldefinidas (cap. XVI) e as doenças do aparelho circulatório (cap. VIII-CID). Nos grupos posteriores, apareceram as doenças do sistema nervoso central e órgãos do sentido (cap. VI-CID), as doenças do aparelho respiratório (cap. VIII-CID) e outras causas, em menor proporção. Observou-se que, com exceção das causas externas, a maioria dos óbitos ocorreu, em maior proporção, entre 10 e 14 anos.

Verificou-se que $41,1 \%$ dos óbitos ocorreram entre adolescentes que estudavam e $28,2 \%$ entre aqueles que exerciam alguma atividade econômica, seja no setor primário (lenheiro, lavrador), secundário (serralheiro, pedreiro, funileiro) ou terciário (balconista, secretária, digitador). Nos anos de altos coeficientes (1990 e 1992), a mortalidade atingiu mais os estudantes no primeiro ano $(50,0 \%$ do total de óbitos), no segundo, mais os trabalhadores $(35,0 \%)$, e os adolescentes sem nenhuma atividade econômica ou classificados como "menor", possivelmente, não estudantes e nem trabalhadores $(35,0 \%)$. Em 1990, a asfixia por afogamento contribuiu com a maior proporção de óbitos $(38,5 \%)$, superando os acidentes de trânsito (30,8\%), enquanto, em 1992, os ferimentos por arma de fogo contribuíram com 48,5\% dos óbitos e acidentes de trânsito com $35,7 \%$.

Considerando apenas as causas externas, observa-se que entre 1984 a 1993 (Tabela 3) a proporção de óbitos foi elevada para o grupo de 15 a 19 anos, $72,6 \%$ contra $27,6 \%$ de 10 a 14 anos e, em ambos grupos, maior para o sexo masculino.

Os acidentes de trânsito, inclusive atropelamento, foram responsáveis por $36,8 \%$ dos óbitos, seguidos por ferimentos por arma de fogo $(29,9 \%)$, asfixia por afogamento $(16,1 \%)$ e quedas $(8,0 \%)$. Em

Tabela 2 - Mortalidade proporcional por causas, segundo grupos etários de 10-19 anos, Município de Botucatu (SP), 1984 a 1993 .

\begin{tabular}{|c|c|c|c|c|c|c|}
\hline \multirow{3}{*}{ Causa } & \multicolumn{6}{|c|}{ Idade } \\
\hline & \multicolumn{2}{|c|}{10 a 14} & \multicolumn{2}{|c|}{15 a 19} & \multicolumn{2}{|c|}{ Total } \\
\hline & $\mathrm{N}^{\mathrm{o}}$ & $\%$ & $\mathrm{~N}^{\circ}$ & $\%$ & $\mathrm{~N}^{\circ}$ & $\%$ \\
\hline Causas externas & 24 & 22,6 & 63 & 72,3 & 87 & 70,2 \\
\hline Neoplasmas & 4 & 57,1 & 3 & 42,8 & 7 & 5,6 \\
\hline Sinais, Sint. e Afec. maldefinidas & 3 & 50,0 & 3 & 50,0 & 6 & 4,8 \\
\hline D. Sist. nervoso e órgãos dos sentidos & 4 & 80,0 & 1 & 20,0 & 5 & 4,0 \\
\hline D. aparelho circulatório & 4 & 66,7 & 2 & 33,3 & 6 & 4,8 \\
\hline D. aparelho respiratório & 3 & 75,0 & 1 & 25,0 & 4 & 3,2 \\
\hline Demais & 3 & 33,3 & 6 & 66,7 & 9 & 7,3 \\
\hline Total & 45 & 36,3 & 79 & 63,7 & 124 & 100,0 \\
\hline
\end{tabular}


Tabela 3 - Distribuição dos óbitos de adolescentes, segundo grupos etários, sexo e causas externas, Município de Botucatu (SP), 1984 a 1993.

\begin{tabular}{|c|c|c|c|c|c|c|c|c|}
\hline \multirow{3}{*}{ Causa } & \multicolumn{8}{|c|}{ Idade } \\
\hline & \multicolumn{3}{|c|}{10 a 14} & \multicolumn{3}{|c|}{15 a 19} & \multicolumn{2}{|c|}{ T.Geral } \\
\hline & Masc. & Fem. & Total & Masc. & Fem. & Total & $\mathrm{N}^{\circ}$ & $\%$ \\
\hline Acidente de trânsito & 5 & 3 & 8 & 20 & 4 & 25 & 32 & 36,8 \\
\hline Ferim. arma de fogo & 4 & & 4 & 20 & 2 & 24 & 26 & 30,0 \\
\hline Ferim. arma branca & & & & 3 & 1 & 4 & 4 & 4,6 \\
\hline Asfixia/afogam. & 7 & 1 & 8 & 5 & 1 & 6 & 14 & 16,1 \\
\hline Queda & 1 & & 1 & 5 & 1 & 6 & 7 & 8,0 \\
\hline Picada de abelha & 2 & & 2 & & & & 2 & 2,3 \\
\hline Acidente de trem & 1 & & 1 & & & & 1 & 1,1 \\
\hline Choque anestésico & & & & & 1 & & 1 & 1,1 \\
\hline \multirow[t]{2}{*}{ Total } & 20 & 4 & 24 & 53 & 10 & 63 & \multirow[t]{2}{*}{87} & \multirow[t]{2}{*}{100,0} \\
\hline & & $27,6 \%$ & & & $72,4 \%$ & & & \\
\hline
\end{tabular}

Tabela 4 - Distribuição dos óbitos de adolescentes por causas externas e ano, Município de Botucatu (SP), 1984 a 1993.

\begin{tabular}{|c|c|c|c|c|c|c|c|c|c|c|}
\hline \multirow{2}{*}{ Ano } & \multicolumn{8}{|c|}{ Causa Externa } & \multicolumn{2}{|c|}{ Total } \\
\hline & $\begin{array}{l}\text { Acid. } \\
\text { Trâns. }\end{array}$ & FAF & FAB & $\begin{array}{l}\text { Afoga- } \\
\text { mento }\end{array}$ & $\begin{array}{l}\text { Acid. } \\
\text { Trem }\end{array}$ & Queda & $\begin{array}{l}\text { Picada } \\
\text { abelha }\end{array}$ & $\begin{array}{c}\text { Choque } \\
\text { anest. }\end{array}$ & $\mathrm{N}^{\mathrm{o}}$ & $\%$ \\
\hline 1984 & 2 & 1 & & & & & & & 3 & 70,0 \\
\hline 1985 & 6 & & 1 & & & 1 & & & 8 & 57,1 \\
\hline 1986 & 1 & 3 & 2 & 4 & & & & & 10 & 76,9 \\
\hline 1987 & 2 & & & & & 2 & & 1 & 5 & 71,4 \\
\hline 1988 & 4 & & & & & & & & 4 & 57,1 \\
\hline 1989 & 1 & 1 & 1 & 1 & & 1 & & & 5 & 50,0 \\
\hline 1990 & 4 & 3 & & 5 & & 1 & & & 13 & 72,2 \\
\hline 1991 & 3 & 7 & & & 1 & & 2 & & 13 & 81,3 \\
\hline 1992 & 5 & 6 & & 2 & & 1 & & & 14 & 70,0 \\
\hline 1993 & 4 & 5 & & 2 & & 1 & & & 12 & 80,0 \\
\hline \multirow[t]{2}{*}{ Total } & 32 & 26 & 4 & 14 & 1 & 7 & 2 & 1 & 87 & \\
\hline & $36,8 \%$ & $29,9 \%$ & $4,6 \%$ & $16,1 \%$ & $2,1 \%$ & $8,0 \%$ & $2,3 \%$ & $1,1 \%$ & & 100,0 \\
\hline
\end{tabular}

FAF - Ferimentos por arma de fogo

FAB - Ferimento por arma branca

todas as causas houve um predomínio do sexo masculino.

Analisando a evolução das diferentes causas externas, no Município de Botucatu, Tabela 4, observou-se que a participação das causas externas no total de óbitos variou de 50,0\% a 81,3\%, entre 1984 a 1993. A partir da década de 1990 , esta participação manteve-se acima de 70,0\% dos óbitos, ocorrendo um padrão mais regular de ocorrência, além dos acidentes de trânsito, de ferimentos por armas de fogo e asfixia por afogamento.

\section{DISCUSSÃO E CONCLUSÕES}

O trabalho revela dados semelhantes aos encontrados na literatura ${ }^{2,4,12}$, ou seja, a mortalidade na fase da adolescência é mais elevada no sexo masculino e no subgrupo de 15 a 19 anos, devido às causas externas. Comparando-se, para o ano de 1985, com os dados encontrados por Lolio e col..$^{12}$, observou-se que o coeficiente de mortalidade por causas externas, em Botucatu, (52,5/100.000) foi maior do que aquele encontrado para o Interior do Estado, (48,5/ 100.000), e metade daquele referente à Capital, (109,5/100.000). Coeficiente semelhante ao de Botucatu, em 1985, apresentou a capital do Estado de São Paulo, em 1977. Em relação à mortalidade proporcional, em 1985, observou-se que o Município de Botucatu apresentou uma proporção de 57,1\% maior do que algumas unidades da Federação como Paraná, 55,1\%, Distrito Federal, 55,6\% e Rio Grande do Sul, $56,6 \%{ }^{12}$.

Como principais causas, foram encontrados os acidentes de trânsito e ferimentos por armas de fogo e asfixia por afogamento, sendo os meninos mais vulneráveis do que as meninas.

Observamos que os sinais, sintomas e afecções maldefinidas (cap. XVI) foram a terceira causa de óbito. Isto pode ser explicado pelo inadequado 
preenchimento dos atestados de óbitos e que, se preenchidos adequadamente, poderiam alterar os resultados de trabalhos retrospectivos como este. Do mesmo modo, uma maior precisão quanto à classificação do "menor", possibilitaria haver melhor caracterização do adolescente. Conseqüentemente, o atestado de óbito, devidamente preenchido, é um elemento importante para se conhecer a tendência da mortalidade por causas e segundo diferentes grupos de adolescentes.

Os óbitos por doenças infecto-parasitárias (cap. I-CID) estão, notadamente, excluídos dos atestados do Município de Botucatu, nesta faixa etária, o que pode supor a melhoria das condições habitacionais e um maior acesso aos serviços de saúde, voltados à atenção à criança e à adolescência.

Entre as causas externas, os afogamentos se equiparam aos acidentes de trânsito, o que poderia ser explicado pela própria característica do adolescente, que se considera invulnerável e parcialmente, onipotente.

O quadro de mortalidade de adolescentes, em Botucatu, é semelhante àquele apresentado no Estado e em todo Brasil, onde acidentes de trânsito e homicídios apresentam-se como as principais causas de mortalidade. Em ambos os sexos, a mortalidade por causas externas é mais evidente na faixa de maior idade.

\section{REFERÊNCIAS BIBLIOGRÁFICAS}

1. BLUM, R.W. Global trends in adolescent health. JAMA, 265 2711-19, 1991.

2. CHOR, D. et al. Diferencial de mortalidade em homens e mulheres em localidades da região Sudeste, Brasil, 1960 , 1970, 1980, Rev. Saúde Pública, 26: 246-55, 1992.

3. CENTRO BRASILEIRO PARA INFÂNCIA E ADOLESCÊNCIA (CBIA). Estatuto da criança e do adolescente: Lei $n^{\circ}$ 8069, 13 de julho de 1990; artigo 2. Brasília, Ministério de Ação Social, 1990.

4. FREITAS, C.B.P.B. \& FREITAS, C.B.D.B. O adolescente e a realidade brasileira. In: Maakaraun, M.F. et al. Tratado de adolescência. Rio de Janeiro, Cultura Médica, 1991. p. 9-30.

5. FUNDAÇÃO IBGE. Censo demográfico: 1970, v. 1, t. 18, $2^{\mathrm{a}}$ parte ( $7^{\circ}$ Recenseamento geral do Brasil, 1970).

6. FUNDAÇÃO IBGE. Sinopse preliminar do censo demográfico: São Paulo. Rio de Janeiro, 1971. (7º Recenseamento geral do Brasil, 1970).

7. FUNDAÇÃO IBGE. Sinopse preliminar do censo demográfico: São Paulo, 1980. Rio de Janeiro, v. 1, t. 1, $\mathrm{n}^{\circ}$ 18. ( $9^{\circ}$ Recenseamento geral do Brasil, 1980).

8. FUNDAÇÃO IBGE. Censo demográfico, 1980, v. 1, t. 3, nº 17. ( $9^{\circ}$ Recenseamento geral do Brasil, 1980).
O presente trabalho confirma os dados nacionais, mostrando que a mortalidade dos adolescentes numa cidade média do interior é semelhante àquela apresentada pelos adolescentes nos grandes centros urbanos do País. Cabe, contudo, investigar, além das características dos adolescentes, as mudanças socioeconômicas, inclusive comportamentais, nas cidades interioranas que propiciaram um maior risco para este grupo etário. Este conhecimento torna-se indispensável para o estabelecimento de programas de saúde, destinados a este grupo etário e a suas famílias, e necessidade de uma maior divulgação nos meios de comunicação, principalmente, considerando que a grande proporção dos óbitos deve-se às causas evitáveis e preveníveis e atinge um grupo que, como assinala Henriques e col. ${ }^{10}$, é "o potencial futuro de uma sociedade, ainda que o fato de ser adolescente, com seu "pensamento mágico", seja o seu maior risco".

\section{AGRADECIMENTOS}

À Monica Kron do Setor de Estatística do Centro de Saúde Escolar, pela codificação das causas de óbitos, e à Eliana Lovizuto Iessi e Maria de Lourdes Silveira, da Faculdade de Medicina de Botucatu (UNESP), pela tabulação dos dados.

9. FUNDAÇÃO IBGE. Censo demográfico, 1991. São Paulo, 1993

10. HENRIQUES, M.H. et al. Adolescentes de hoje, pais de amanhã: Brasil. New York, The Alan Guttmacher Institute, s.d.

11. LEBRÃO, M.L. Utilização da Classificação Internacional de Doenças: informações de morbidade. São Paulo, Centro da OMS para Classificação de Doenças em Português/ Núcleo de Estudos em População e Saúde, 1993. (Série Divulgação, 8).

12. LOLIO,C.A. et al. Mortalidade de adolescentes no Brasil, 1977,1980,1985 - Magnitudes e tendências. Rev. Saúde Pública, 24: 481-90,1990.

13. ORGANIZAÇÃO MUNDIAL DE SAÚDE. Manual de classificação estatística internacional de doenças, lesões e causas de óbitos. $9^{\circ}$ Revisão, 1975. São Paulo, Centro Brasileiro de Classificação de Doenças, 1979. v.1

14. WORLD HEALTH ORGANIZATION STUDY GROUP ON YOUNG PEOPLE AND HEALTH FOR ALL BY THE YEAR 2000, Geneva, 1984. Report. Geneva, 1986. (WHO Techn. Rep. Ser., 731). 